\title{
Calculation of displacement cross sections at intermediate and high energies of primary particles using results of molecular dynamics simulations
}

\author{
A.Yu. Konobeyeva , C.H.M. Broeders, and U. Fischer \\ Institute for Reactor Safety, Forschungszentrum Karlsruhe, Postfach 3640, 76021 Karlsruhe, Germany
}

\begin{abstract}
A method combining the intranuclear cascade evaporation model, the binary collision approximation model and the method of molecular dynamics is proposed for the calculation of displacement cross sections for structural materials irradiated with intermediate and high energy particles. Calculated displacement cross sections are compared with experimental data for copper irradiated with 1.1 and $1.94 \mathrm{GeV}$ protons. Calculations up to the proton energy $0.1 \mathrm{TeV}$ are discussed.
\end{abstract}

\section{Introduction}

Materials of advanced nuclear energy systems as the fusion reactor and ADS are considered to be irradiated by extremely intense fluxes of energetic particles. The study of the radiation damage of structural materials for these facilities relies on the accurate calculation of displacement cross sections at intermediate and high energies.

The NRT model [1] is frequently used for the calculation of the damage accumulation in irradiated materials. The relative simplicity of the approach provides its use in widespread codes such as NJOY, MCNPX, and others. Available experimental data [2] and more rigorous calculations show the difference with predictions of the NRT model that makes questionable its use for reliable calculations of the displacement cross section and the radiation damage rate.

In the present work the number of defects produced by primary knock on atoms (PKA) in materials is calculated with the help of the binary collision approximation model (BCA) using the results obtained by the method of the molecular dynamics (MD). Calculations of primary recoil spectra are performed using various nuclear models, including the optical model and different versions of intranuclear cascade evaporation model. mula

The displacement cross section is calculated by the for-

$$
\sigma_{d}\left(E_{p}\right)=\sum_{i} \int_{\mathrm{E}_{\mathrm{d}}}^{T_{\mathrm{i}}^{\max }} \frac{d \sigma\left(\mathrm{E}_{\mathrm{p}}, \mathrm{T}_{\mathrm{i}}, Z_{\mathrm{T}}, \mathrm{A}_{\mathrm{T}}, Z_{\mathrm{i}}, A_{\mathrm{i}}\right)}{d T_{\mathrm{i}}} v\left(T_{\mathrm{i}}\right) d T_{\mathrm{i}},
$$

where $E_{p}$ is the incident particle energy; $d \sigma / d T_{i}$ is the differential cross section of the energy transfer to the recoil atom; $\mathrm{Z}_{\mathrm{i}}$ and $\mathrm{A}_{\mathrm{i}}$ are the atomic number and the mass number of the recoil atom, correspondingly; $\mathrm{Z}_{\mathrm{T}}$ and $\mathrm{A}_{\mathrm{T}}$ are the same for the target material; $v\left(\mathrm{~T}_{\mathrm{i}}\right)$ is the number of Frenkel pairs produced by PKA with the kinetic energy $\mathrm{T}_{\mathrm{i}}, \nu\left(\mathrm{T}_{\mathrm{i}}\right)=\eta \cdot \mathrm{N}_{\mathrm{NRT}}$, where $\mathrm{N}_{\mathrm{NRT}}$ is the number of defects predicted by NRT [1]: $\mathrm{N}_{\mathrm{NRT}}=0.8 \cdot \mathrm{T}_{\mathrm{dam}} /\left(2 \mathrm{E}_{\mathrm{d}}\right) ; \mathrm{T}_{\mathrm{dam}}$ is the damage energy [1]; $\eta$ is the defect production efficiency [2]; $\mathrm{E}_{\mathrm{d}}$ is effective threshold displacement energy; $\mathrm{T}_{\mathrm{i}}^{\max }$ is the maximal kinetic energy of

\footnotetext{
${ }^{a}$ Presenting author,e-mail: konobeev@irs.fzk . de
}

the PKA produced in i-th reactions; the summation is over all recoil atoms produced in the irradiation.

\section{Calculation of the number of defects produced in irradiated materials}

The interaction of high energy nucleons with materials produces recoil atoms with kinetic energies considerably exceeding the present maximum energy of MD simulations $[3,4]$.

For an energetic ion moving in the material the simulation of the atomic collision was performed by BCA up to a certain "critical" energy ( $\mathrm{T}_{\text {crit }}$ ) of the ion. Below this energy the BCA calculation was stopped and the number of defects has been evaluated according to the results of the MD simulation. This procedure was performed for all PKAs produced in the atomic collision cascades.

An example of such calculations is presented below for copper. The number of Frenkel pairs created by ions with the energy below $\mathrm{T}_{\text {crit }}$ has been estimated according to the empirical equation [5], which approximates results of the MD simulation: $\eta=0.7066 T_{\text {dam }}^{-0.437}+2.28 \times 10^{-3} T_{\text {dam }}$, where $\mathrm{T}_{\text {dam }}$ is in $\mathrm{keV}$. The $\mathrm{T}_{\text {crit }}$ value corresponds to the damage energy $\mathrm{T}_{\mathrm{dam}}$ equal to $20 \mathrm{keV}$ and the defect production efficiency $\eta$ equal to 0.24 [5]. For the self-ion irradiation of copper $\mathrm{T}_{\text {crit }}$ is equal to $28.3 \mathrm{keV}$.

The ratio of calculated number of Frenkel pairs to $v(\mathrm{~T})$ predicted by the NRT model (efficiency of the defect generation) is shown in figure 1 for the $\mathrm{Cu}-\mathrm{Cu}$ irradiation. The value of the efficiency $\eta$ is shown as a function of the damage energy $\mathrm{T}_{\mathrm{dam}}$ in the energy range which corresponds to the primary kinetic energy of $\mathrm{Cu}$-ions up to $5.0 \mathrm{GeV}$. The value of $\mathrm{E}_{\mathrm{d}}$ adopted for copper is equal to $30 \mathrm{eV}$ [2]. Calculations were performed using the IOTA code [7].

Apparently, the increase of the efficiency at high energies, $\mathrm{T}_{\mathrm{dam}}$ above $20 \mathrm{keV}$ (fig. 1) results from the growth of the number of atomic collisions with relatively small energies transferred from the projectile to lattice ions with the increase of the projectile energy. Small energies transferred to PKAs correspond to the region with high values of the defect production efficiency. 


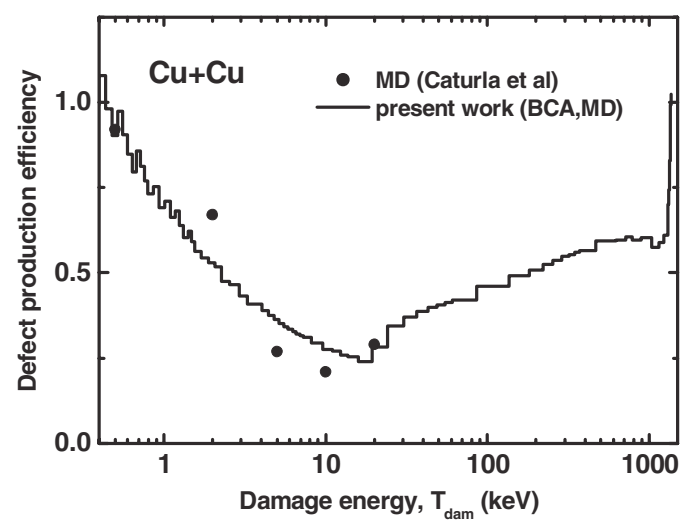

Fig. 1. The efficiency of the defect production for the $\mathrm{Cu}-\mathrm{Cu}$ irradiation obtained using the combined BCA-MD method (histogram) and results of the MD simulation [5] (dots).

\section{Displacement cross sections for elastic proton scattering}

Generally, the spectrum of PKAs produced by the proton elastic scattering includes the contributions from the screened Coulomb scattering in material, the nuclear scattering and their interference.

At low incident energies the screening effect plays an important role in the proton elastic scattering on atoms. The differential cross section for the energy transfer from proton to the lattice atom can be written in the following form

$$
\mathrm{d} \sigma\left(\mathrm{E}_{\mathrm{p}}, \mathrm{T}\right)=\pi \mathrm{a}^{2} \mathrm{f}\left(\mathrm{t}^{1 / 2}\right) \frac{\mathrm{dt}}{2 \mathrm{t}^{3 / 2}}
$$

where the $\mathrm{f}\left(\mathrm{t}^{1 / 2}\right)$ is the screening function, " $\mathrm{a}$ " is the screening length, and " $\mathrm{t}$ " is the reduced energy $[6,7]$.

The screening effect disappears with the increase of the incident proton energy. For the proton energy above $1 \mathrm{MeV}$ the difference between the elastic displacement cross section $\left(\sigma_{\text {d,el }}\right)$ calculated by equation (2) with $\mathrm{f}\left(\mathrm{t}^{1 / 2}\right)$ from ref. [6] and $\sigma_{\text {d,el }}$ estimated using the Rutherford formula is less than $2.5 \%$ for $\mathrm{Al},<4.3 \%$ for $\mathrm{Cu}$ and $<6.5 \%$ for $\mathrm{W}$.

The contribution of the nuclear scattering in the recoil spectrum $\mathrm{d} \sigma / \mathrm{dT}$ increases with the increase of the primary proton energy. The contribution becomes appreciable for the $\sigma_{\text {d,el }}$ calculation at energies above $\sim 5 \mathrm{MeV}$, where the screening effect is small. It allows applying the nuclear optical model for the elastic displacement cross section calculations.

Figure 2 shows the proton elastic displacement cross section for copper at the energy range from $\sim 1 \mathrm{keV}$ up to $100 \mathrm{GeV}$. The differential cross section for the energy transfer to PKA $\mathrm{d} \sigma\left(\mathrm{E}_{\mathrm{p}}, \mathrm{T}\right)$ has been calculated using equation (2) with $f\left(t^{1 / 2}\right)$ from ref. [6] for the proton energy up to $1 \mathrm{MeV}$, optical model with parameters from [8] and [9] up to $E_{p}=0.4 \mathrm{GeV}$ and using relativistic formula from ref. [10] (corrected). The number of defects was calculated using results of combined BCA-MD simulations (sect. 2) and the NRT model.

The increase of $\sigma_{\text {d,el }}$ at the energy above $1 \mathrm{GeV}$ is due to the relativistic effect. The classical Rutherford formula predicts a sharp decline of the cross section at these energies.

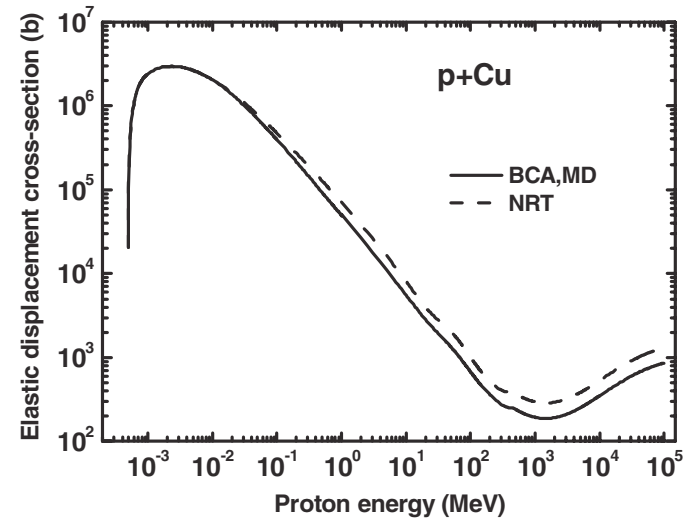

Fig. 2. Displacement cross section for elastic proton interactions with natural copper calculated using the BCA-MD method (solid line) and the NRT model (dashed line).

Table 1. Displacement cross section (b) for nonelastic proton interactions with ${ }^{63} \mathrm{Cu}$ at $20 \mathrm{MeV}$ incident energy obtained using various nuclear models for calculating recoil spectra, the BCA-MD approach, and the NRT model. The nonelastic cross section for $\mathrm{p}+{ }^{63} \mathrm{Cu}$ is equal to $1.12 \mathrm{~b}$.

\begin{tabular}{lcc}
\hline Nuclear model & BCA-MD & NRT \\
\hline Bertini/Dresner & 1430 & 2600 \\
Bertini/ABLA & 1520 & 2760 \\
ISABEL/Dresner & 1420 & 2570 \\
ISABEL/ABLA & 1470 & 2670 \\
CEM03 & 1380 & 2550 \\
CASCADE & 1640 & 2890 \\
DISCA-C & 2020 & 3480 \\
Average value & $1550 \pm 220$ & $2790 \pm 330$ \\
\hline
\end{tabular}

\section{Displacement cross sections for nonelastic proton interactions with nuclei}

To get the displacement cross section for nonelastic particle interactions with atoms $\left(\sigma_{\mathrm{d}, \text { non }}\right)$ collision cascades were simulated for all residual atoms produced in the nuclear reaction using the method described above.

Tables 1-3 show displacement cross sections for the proton nonelastic interaction with ${ }^{63} \mathrm{Cu}$ at various incident energies. Calculations of recoil energy distributions were performed using various nuclear models implemented in MCNPX [11], CASCADE [12], and DISCA-C [13] codes. The number of defects has been calculated by the BCA-MD method and by the NRT model.

One may note the $\sigma_{\mathrm{d} \text {,non }}$ value for ${ }^{63} \mathrm{Cu}$ from $\mathrm{ENDF} / \mathrm{B}$ VI.8 obtained using the NRT model is equal to $3472 \mathrm{~b}$ for the proton incident energy $20 \mathrm{MeV}$ and $3715 \mathrm{~b}$ for $\mathrm{E}_{\mathrm{p}}=150 \mathrm{MeV}$. It is close to the DISCA-C calculation (tables 1 and 2).

The calculation of $\sigma_{\mathrm{d} \text {,non }}$ for copper shows that the nonelastic displacement cross section can be obtained using the efficiency of the defect generation $\eta\left(\mathrm{T}_{\mathrm{dam}}\right)$ calculated for $\mathrm{Cu}-\mathrm{Cu}$ irradiation (fig. 1) for all residual atoms formed in the nuclear reaction $\mathrm{p}+\mathrm{Cu}$. It gives a possibility to perform a quick evaluation of $\sigma_{\mathrm{d}, \text { non }}$ avoiding time consuming simulations. The difference between the estimated value of $\sigma_{\mathrm{d} \text {,non }}$ and the result of detailed modeling increases with the energy of primary 
Table 2. Displacement cross section (b) for nonelastic proton interactions with ${ }^{63} \mathrm{Cu}$ at $150 \mathrm{MeV}$ incident energy calculated using various nuclear models, the BCA-MD approach, and the NRT model. The proton nonelastic cross section is equal to $0.734 \mathrm{~b}$.

\begin{tabular}{lcc}
\hline Nuclear model & BCA-MD & NRT \\
\hline Bertini/Dresner & 1790 & 3060 \\
Bertini/ABLA & 1890 & 3230 \\
ISABEL/Dresner & 1730 & 2950 \\
ISABEL/ABLA & 1820 & 3110 \\
CEM03 & 1780 & 3050 \\
INCL4/Dresner & 1920 & 3260 \\
INCL4/ABLA & 2010 & 3410 \\
FLUKA/Dresner & 2400 & 4050 \\
FLUKA/ABLA & 2510 & 4220 \\
CASCADE & 1740 & 3000 \\
DISCA-C & 2070 & 3500 \\
Average value & $1970 \pm 270$ & $3350 \pm 430$ \\
\hline
\end{tabular}

Table 3. Displacement cross section (b) for nonelastic proton interactions with ${ }^{63} \mathrm{Cu}$ at $50 \mathrm{GeV}$ incident energy calculated using various nuclear models, the BCA-MD approach, and the NRT model. The proton nonelastic cross section is equal to $0.791 \mathrm{~b}$.

\begin{tabular}{lcc}
\hline Nuclear model & BCA-MD & NRT \\
\hline FLUKA/Dresner & 930 & 1360 \\
FLUKA/ABLA & 1390 & 2130 \\
CASCADE & 1420 & 2520 \\
Average value & $1250 \pm 270$ & $2000 \pm 590$ \\
\hline
\end{tabular}

Table 4. Total displacement cross section (b) (the sum of elastic and nonelastic components) for natural copper irradiated with $1.1 \mathrm{GeV}$ protons. The elastic displacement cross sections $\sigma_{\mathrm{d} \text {,el }}$ calculated by BCA-MD is equal to $191.3 \mathrm{~b}$ and by NRT is $291.9 \mathrm{~b}$. The measured $\sigma_{\mathrm{d}}$ value is equal to $1440 \mathrm{~b}$ [14].

\begin{tabular}{lcc}
\hline Model & BCA-MD & NRT \\
\hline Bertini/Dresner & 2170 & 3890 \\
Bertini/ABLA & 2360 & 4260 \\
CEM03 & 2180 & 3890 \\
INCL4/Dresner & 2590 & 4620 \\
INCL4/ABLA & 2750 & 4920 \\
FLUKA/Dresner & 2790 & 5190 \\
FLUKA/ABLA & 3090 & 5790 \\
CASCADE & 2290 & 4140 \\
Average value & $2530 \pm 330$ & $4590 \pm 680$ \\
$\sigma_{\mathrm{d}}($ average $) / \sigma_{\mathrm{d}}(\exp )$ & 1.76 & 3.19 \\
\hline
\end{tabular}

protons. It is about $1-11 \%$ for the proton energy $1 \mathrm{GeV}$, depending on the model, and $25-33 \%$ at $\mathrm{E}_{\mathrm{p}}=100 \mathrm{GeV}$.

\section{Comparison with experimental data}

Experimental data for copper are available at the proton incident energies 1.1 and $1.94 \mathrm{GeV}$ [14]. At low proton energies there are data derived by Jung [15] from experimental electron, light ion, and neutron damage rates.

Tables 4 and 5 show the total displacement cross section $\left(\sigma_{\mathrm{d} \text {,el }}+\sigma_{\mathrm{d} \text {,non }}\right)$ calculated for copper irradiated with 1.1 and
Table 5. Total displacement cross section (b) (the sum of elastic and nonelastic components) for natural copper irradiated with $1.94 \mathrm{GeV}$ protons. The elastic displacement cross sections $\sigma_{\mathrm{d}, \mathrm{el}}$ calculated by BCA-MD is equal to $191.4 \mathrm{~b}$ and by NRT is $291.8 \mathrm{~b}$. The measured $\sigma_{\mathrm{d}}$ value is equal to $1830 \mathrm{~b}$ [14].

\begin{tabular}{lcc}
\hline Model & BCA-MD & NRT \\
\hline Bertini/Dresner & 1940 & 3420 \\
Bertini/ABLA & 2170 & 3870 \\
CEM03 & 1880 & 3270 \\
INCL4/Dresner & 2510 & 4470 \\
INCL4/ABLA & 2660 & 4760 \\
FLUKA/Dresner & 2550 & 4710 \\
FLUKA/ABLA & 2860 & 5380 \\
CASCADE & 2210 & 3970 \\
Average value & $2350 \pm 350$ & $4230 \pm 730$ \\
$\sigma_{\mathrm{d}}($ average $) / \sigma_{\mathrm{d}}(\exp )$ & 1.28 & 2.31 \\
\hline
\end{tabular}

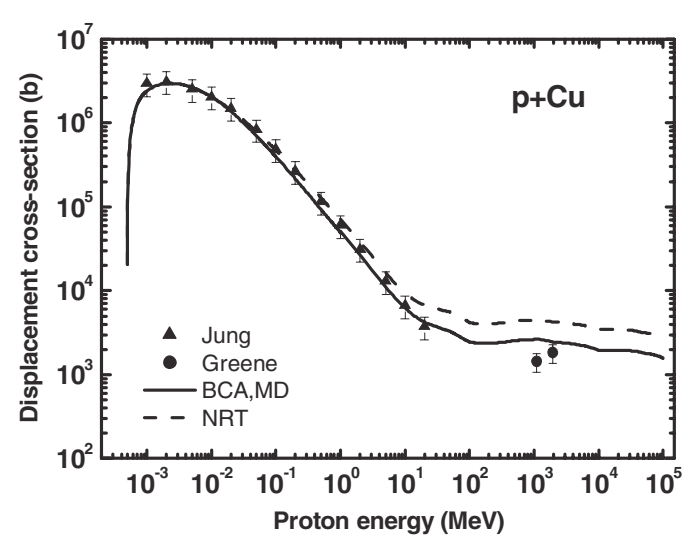

Fig. 3. Total displacement cross section $\left(\sigma_{\mathrm{d}, \mathrm{el}}+\sigma_{\mathrm{d} \text {,non }}\right)$ for the proton irradiation of copper calculated using the BCA-MD approach (solid line) and the NRT model (dashed line), displacement cross section obtained by Jung [15] from the analysis of various experimental data (triangle) and data measured in ref. [14] (circle).

Table 6. Ratio of the total displacement cross section for copper calculated by BCA-MD and NRT.

\begin{tabular}{lc}
\hline Proton energy & $\sigma_{\mathrm{d}}($ BCA-MD $) / \sigma_{\mathrm{d}}($ NRT $)$ \\
\hline $10 \mathrm{keV}$ & 1.0 \\
$100 \mathrm{keV}$ & 0.836 \\
$1 \mathrm{MeV}$ & 0.700 \\
$10 \mathrm{MeV}$ & 0.655 \\
$100 \mathrm{MeV}$ & 0.588 \\
$1 \mathrm{GeV}$ & 0.585 \\
$10 \mathrm{GeV}$ & 0.563 \\
$100 \mathrm{GeV}$ & 0.570 \\
\hline
\end{tabular}

$1.94 \mathrm{GeV}$ protons. The elastic component of the cross section has been obtained as described in section 3 . The displacement cross section for nonelastic proton interactions has been calculated using various nuclear models implemented in codes from refs. [11-13]. The BCA-MD approach and the NRT model were used for the calculation of the number of defects produced in the material by PKAs formed in nuclear reactions.

There is a big difference between measured displacement cross sections and $\sigma_{\mathrm{d}}$ calculated using the NRT model. 
The agreement with BCA-MD calculations is better. Predictions of Bertini, CEM03 and CASCADE models combined with BCA-MD are best.

Figure 3 shows the total displacement cross section obtained for copper at proton incident energies from several $\mathrm{keV}$ to $100 \mathrm{GeV}$. Displacement cross sections for nonelastic proton interactions included in $\sigma_{\mathrm{d}}$ were obtained by averaging of $\sigma_{\text {d,non }}$ values calculated using different nuclear models. Models suitable for the $\sigma_{\mathrm{d} \text {,non }}$ calculation at various proton energies are different. Examples of appropriate models used for calculations at various protons energies are shown in tables 1-3.

One should note that the use of Bertini, CEM, and ISABEL models at proton energies $<100 \mathrm{MeV}$ is justified by their use in the combination with the pre-equilibrium exciton model.

Table 6 shows ratios of the displacement cross section calculated for copper using the BCA-MD approach and the NRT model.

\section{Conclusion}

Displacement cross sections obtained using the intranuclear cascade evaporation model, the binary collision approximation model and results of molecular dynamics simulations have been presented for copper. The resulting displacement cross sections are in better agreement with available experimental data than cross sections calculated on the basis of the NRT model.

\section{References}

1. M.J. Norgett, M.T. Robinson, I.M. Torrens, Nucl. Eng. Des. 33, 50 (1975).

2. C.H.M. Broeders, A.Yu. Konobeyev, J. Nucl. Mater. 328, 197 (2004).

3. C.H.M. Broeders, A.Yu. Konobeyev, J. Nucl. Mater. 336, 201 (2005).

4. C.H.M. Broeders, A.Yu. Konobeyev, C. Villagrasa, J. Nucl. Mater. 342, 68 (2005).

5. M.J. Caturla, T. Diaz de la Rubia, M. Victoria et al., J. Nucl. Mater. 296, 90 (2001).

6. K.B. Winterbon, P. Sigmund, J.B.K. Sanders, K. Dan, Vidensk. Selsk. Mat. Fys. Medd. 37, N14, 1 (1970).

7. C.H.M. Broeders, A.Yu. Konobeyev, K. Voukelatou, FZKA 6984, 2004; http://bibliothek.fzk.de/zb/berichte/FZKA6984.pdf.

8. A.J. Koning, J.-P. Delaroche, Nucl. Phys. A 713, 231 (2003).

9. D.G. Madland, in Proc. OECD/NEA Spec. Meeting on Nucleon Nucleus Opt. Mod. up to $200 \mathrm{MeV}$, Bruyer̀es-le-Châtel, 1996, p. 129; http://www.nea.fr/html/science/om200/.

10. Insoo Jun, IEEE Trans. Nucl. Sci. 48, 162 (2001).

11. J.S. Hendricks, G.W. McKinney, J.W. Durkee et al., LA-UR-067991 Dec. 7, 2006.

12. V.S. Barashenkov, Comp. Phys. Commun. 126, 28 (2000).

13. C.H.M. Broeders, A.Yu.Konobeyev, Yu.A. Korovin, V.N. Sosnin, Forschungszentrum Karlsruhe Report FZKA 7221, June 2006, http://bibliothek.fzk.de/zb/berichte/ FZKA7221.pdf.

14. G.A. Greene, C.L. Snead Jr., C.C. Finfrock et al., in Proc. 6th Int. Meet. Nucl. Appl. Acc. Technol., Illinois, 2004, p. 881.

15. P. Jung, J. Nucl. Mater. 117, 70 (1983). 